\title{
Cuidados paliativos no âmbito hospitalar: compreensão de enfermeiros
}

\author{
Palliative care for hospital environments as a nursing picture: understanding by nurses \\ Cuidados paliativos para ambientes hospitalarios como un cuadro de enfermería: \\ comprensión por enfermeros
}

\begin{abstract}
Larissa Leal Lopes ${ }^{1}$, Patrícia Serpa de Souza Batista ${ }^{1}$, Débora Rodrigues Alves de Lima ${ }^{1 *}$, Amanda Maritsa de Magalhães Oliveira ${ }^{1}$, Kalina Coeli da Costa ${ }^{1}$.
\end{abstract}

\section{RESUMO}

Objetivo: investigar a compreensão de enfermeiros acerca dos cuidados paliativos a pacientes assistidos no âmbito hospitalar. Método: estudo do tipo exploratório, com abordagem qualitativa, realizado no período de fevereiro a março de 2018, mediante entrevista semi-estruturada com 20 enfermeiros assistenciais de um hospital universitário. Os dados foram submetidos a análise de conteúdo de Bardin. Resultados: da análise do material empírico emergiram quatro categorias: A compreensão dos enfermeiros sobre cuidados paliativos; Prática assistencial promovida pelos enfermeiros ao cuidar de pacientes em cuidados paliativos; Relacionamento entre pacientes em cuidados paliativos, equipe multiprofissional e familiares; Dificuldades encontradas pelos enfermeiros na assistência direcionada ao paciente em cuidados paliativos. Conclusão: foi possível compreender a importância dos cuidados paliativos no âmbito hospitalar na visão dos enfermeiros, tornandose necessário e fundamental a educação permanente específica destes profissionais para melhoria da qualidade da assistência prestada ao paciente e sua família.

Palavras-chave: Enfermagem, Cuidados Paliativos, Assistência ao paciente.

\begin{abstract}
Objective: to investigate the understanding by nurses about palliative cared at hospital environment for patients assisted in the hospital setting. Method: exploratory study, with a qualitative approach, carried out from February to March 2018, through a semi-structured interview with 20 nursing assistants from a university hospital. The data were submitted to Bardin content analysis. Results: from the analysis of the empirical material emerged four categories: Nurses' understanding of palliative care; Nursing practice promoted by nurses when caring for patients in palliative care; Relationship between patients in palliative care, multiprofessional team and family; Difficulties encountered by nurses in patients care in palliative care.
\end{abstract}

Conclusion: it was possible to understand the importance of hospital palliative care in the nurses' view, making it necessary and fundamental the specific permanent education of these professionals, in order to improve the quality of care provided to the patient and his / her family.

Keywords: Nursing, Palliative Care, Patient Care.

\section{RESUMEN}

Objetivos: investigar la comprensión de enfermeros acerca de los cuidados paliativos a pacientes asistidos en el ámbito hospitalario. Método: estudio del tipo exploratorio, con abordaje cualitativo, realizado en el período de febrero a marzo de 2018, mediante entrevista semiestructurada con 20 enfermeros asistenciales de un hospital universitario. Los datos se sometieron al análisis de contenido de Bardin. Resultados: de la

1Universidade Federal da Paraíba (UFPB), João Pessoa - Paraíba. *E-mail: deboraufpbsud@gmail.com 
análisis del material empírico surgieron cuatro categorías: La comprensión de los enfermeros sobre cuidados paliativos; Práctica asistencial promovida por los enfermeros al cuidar de pacientes en cuidados paliativos; Relación entre pacientes en cuidados paliativos, equipo multiprofesional y familiares; Dificultades encontradas por los enfermeros en la asistencia dirigida al paciente en cuidados paliativos. Conclusión: fue posible comprender la importancia de los cuidados paliativos en el ámbito hospitalario en la visión de los enfermeros, tornándose necesario y fundamental la educación permanente específica de estos profesionales para mejorar la calidad de la asistencia prestada al paciente y su familia.

Palabras clave: Enfermería, Cuidados Paliativos, Atención al Paciente.

\section{INTRODUÇÃO}

O cuidado é o cerne da atuação profissional da enfermagem, constituindo-se como tema central da profissão (QUEIRÓS PJP, 2015; LIMA MPO, OLIVEIRA MCX, 2015). No campo da enfermagem os cuidados devem ser ofertados a todos que precisam, desde o nascimento até a morte (FELIX ZC, et al., 2014).

Surge assim, o cuidado paliativo como uma forma de assistência que permite aos profissionais cuidarem de forma prudente e cautelosa; visto que, estão prestando cuidados à paciente cuja progressão da enfermidade ameaça a vida. Tendo o objetivo de proporcionar uma melhor qualidade de vida a pacientes e familiares, através da prevenção e alívio do sofrimento, da dor e de outros problemas de ordem física, social, psicológica e espiritual (FERNANDES MA, et al., 2013; LIMA MPO, OLIVEIRA MCX, 2015; WHO, 2017).

Os cuidados paliativos são realizados principalmente no âmbito hospitalar e constata-se que $75 \%$ das mortes acontecem nesse ambiente, por ser um local de elevada condição científica e tecnológica. Nessa conjuntura, o enfermeiro é capaz de prestar a assistência utilizando diversos recursos materiais e tecnológicos (ISHIKAWA Y, et al., 2013; VASQUES TCS, 2016).

O processo de modificação no paradigma entre a cura e os cuidados paliativos é algo que necessita da ação dos profissionais que atuam nesses cuidados e das instituições acadêmicas, devendo ofertar uma prática embasada em princípios científicos. Mas, os profissionais devem sempre empoderar-se de conhecimentos nessa linha de cuidado (BALIZA MF, et al., 2015).

Entretanto, ainda existe uma deficiência por parte dos enfermeiros em lidar com esses pacientes em cuidados paliativos; uma vez que a filosofia dos cuidados paliativos não se encontra inserida na rotina de cuidados de muitos profissionais, não estando, portanto, realmente hábeis para atendê-los (BRITTO SMC, et al., 2015).

Com base nesse entendimento, torna-se essencial dominar as perspectivas relacionadas à realização dos cuidados paliativos, assim como, o processo da morte; proporcionando aos enfermeiros conhecer suas crenças e valores diante da morte, devendo os mesmos estar aptos a prestar cuidados aos pacientes e familiares que se encontram nessas circunstancias (SILVEIRA NR, et al., 2016).

Diante das inúmeras demandas apresentadas acima, da necessidade de apoio profissional no cuidado paliativo ao paciente e sua família, da busca de respostas que apontam para a importância desses cuidados e para a melhoria do cuidado em saúde aos pacientes em uma unidade de internação hospitalar, questionou-se: Qual a compreensão dos enfermeiros acerca dos cuidados paliativos na assistência hospitalar? Para responder a essa indagação este estudo teve por objetivo investigar a compreensão de enfermeiros acerca dos cuidados paliativos a pacientes assistidos no âmbito hospitalar.

\section{MÉTODO}

Estudo exploratório com abordagem qualitativa, realizado na unidade de Clínica Médica de um hospital universitário no município de João Pessoa - PB. A população foi composta de 34 enfermeiros assistenciais que prestam cuidados na referida unidade. A amostra foi composta por 20 enfermeiros assistenciais. 
A princípio não foi definido o tamanho da amostra, utilizando-se, para tal, o critério de saturação teórica, concluindo-se a coleta ao verificar-se a repetição de informações obtidas nas falas dos participantes. Para seleção da amostra foram considerados os seguintes critérios de inclusão: que o enfermeiro estivesse em atividade laboral durante o período da coleta de dados, que estivesse no exercício da sua profissão há pelo menos seis meses, e que aceitasse participar da pesquisa proposta. Os critérios de exclusão foram: profissional estar de férias, licença ou possuir algum tipo de afastamento.

A coleta de dados foi realizada no período de fevereiro a março de 2018 , mediante a técnica de entrevista semi-estruturada utilizando um roteiro de entrevistas. O material empírico foi gravado em mídia eletrônica. Os dados empíricos oriundos das entrevistas foram analisados qualitativamente por meio da técnica de Análise de Conteúdo que consiste em três etapas: a primeira diz respeito à pré-análise; a segunda, incide na exploração do material; e a terceira, refere-se ao tratamento e interpretação dos resultados obtidos (BARDIN L, 2011).

A pré-análise é a fase de organização propriamente dita, a qual tem o objetivo de operacionalizar e sistematizar as ideias iniciais; a fase de exploração do material consiste na definição das categorias e da codificação. Os dados brutos são organizados e agrupados em categorias, as quais permitem uma descrição das características pertinentes do material empírico; e a última fase consiste no tratamento e interpretação dos resultados obtidos. Nesse momento, é atribuída significação às características do texto, resumidas após tratamento e apresentadas sob a forma de categorias (BARDIN, 2011).

Referente aos aspectos éticos, o presente estudo foi norteado pelas Diretrizes e Normas Regulamentadoras da pesquisa envolvendo seres humanos dispostas na Resolução 466/2012 do Conselho Nacional de Saúde, principalmente no que concerne aos aspectos éticos da pesquisa envolvendo seres humanos e informações pertinentes à pesquisa contemplada no Termo de Consentimento Livre e Esclarecido (BRASIL, 2012). Para garantir o anonimato dos participantes, utilizou-se a sigla "ENF", seguida do número cronológico da ordem de realização da entrevista. Vale ressaltar que a pesquisa foi iniciada após o projeto ser aprovado pelo Comitê de Ética em Pesquisa, tendo como número do CAAE n80973417.5.0000.5183.

\section{RESULTADOS E DISCUSSÃO}

Participaram do estudo 20 enfermeiros assistenciais que prestam cuidados na referida unidade de Clínica Médica do hospital, localizado no município de João Pessoa - PB. Dos enfermeiros que participaram do estudo, $15(75 \%)$ são do sexo feminino e cinco $(25 \%)$ do sexo masculino. Quanto a faixa etária dos enfermeiros está variou dos 28 aos 61 anos, sendo a faixa etária de 30 a 40 anos a que mais prevaleceu entre os participantes com 15 (75\%). Com relação ao estado civil, 11 (55\%) eram casados, seis (30\%) eram solteiros e três (15\%) eram divorciados. No que concerne as capacitações dos profissionais como residência, especialização, mestrado e doutorado, foi observado que $13(65 \%)$ dos enfermeiros possuem especializações, três (15\%) cursam mestrado, dois (10\%) possuem residência, um (5\%) possui mestrado e um $(5 \%)$ cursa o doutorado.

\section{Categoria I - A compreensão dos enfermeiros sobre Cuidados Paliativos}

Os enfermeiros entrevistados discorreram acerca de sua compreensão sobre os cuidados paliativos, conforme mencionados nos seguintes trechos dos depoimentos:

"[...] cuidados realizados ao paciente em que a conduta médica e terapêutica não condiz mais com a cura, são medidas para prolongar o bem-estar do paciente, garantindo um cuidado terapêutico por toda equipe multiprofissional." (ENF 3)

"O cuidado paliativo pode ser definido como uma assistência prestada por uma equipe multiprofissional, cujo objetivo é oferecer uma qualidade de vida ao paciente quando todos os recursos terapêuticos não foram suficientes para um bom prognóstico, estando em sua fase final de vida. Oferecendo conforto ao paciente e sua família." (ENF 6) 
"É uma assistência voltada a minimizar o sofrimento do paciente, minimizar a dor, proporcionar conforto, tanto ao paciente como a sua família." (ENF 14)

Com base nos depoimentos, foi possível identificar a compreensão dos enfermeiros acerca dos cuidados paliativos, estando voltado para o conforto do paciente e da família, para a qualidade de vida e diminuição da dor. O cuidado paliativo é entendido como cuidado que deve ser prestado buscando ofertar conforto ao paciente, devendo sempre proporcionar alívio da dor e do sofrimento. Eles são realizados por uma equipe multiprofissional para pacientes que possuem uma doença ameaçadora da vida, buscando sempre ofertar uma melhora em sua qualidade de vida nos aspectos sociais, físicos, psicológicos e culturais, dando suporte e amparo a família durante todo esse processo de doença, morte e luto (QUEIROZ TA, et al., 2018).

O entendimento dos participantes sobre cuidados paliativos busca garantir aos pacientes uma morte digna, como verificado a seguir:

"É você deixar de visar apenas a doença, e visar o ser humano e sua família. Tentando diminuir ao máximo as ações intervencionistas para que ele passe de maneira digna esse final de curso de vida."(ENF 5)

"[...] São aqueles cuidados que damos e orientamos o paciente para que ele tenha um processo de morte digna para que ele não sofra, e tenha uma melhor qualidade na progressão das limitações que ele vai apresentando com o tempo." (ENF 17)

"[...]. Os cuidados são destinados a proporcionar bem-estar para que ele possa ter dignidade quando estiver sob nossa assistência, procuramos sempre envolver a família nesse cuidado [...]." (ENF 20)

Conforme expresso nos discursos dos participantes deve-se garantir aos pacientes uma morte digna permitindo que ele passe pelo curso final de sua vida com o mínimo de sofrimento e buscando sempre envolver a família em todo esse processo.

Os enfermeiros relatam sobre a responsabilização pela garantia da dignidade do paciente, visto que eles estão em uma condição de extrema fragilidade, hospitalizado, longe de seu lar e de sua família. Logo, esses cuidados devem garantir a manutenção da dignidade humana em todo curso final e natural da vida. A Academia Nacional de Cuidados Paliativos afirmou que os cuidados paliativos buscam a melhoria da qualidade de vida e a manutenção da dignidade humana no decorrer da doença, morte e período de luto (ANCP, 2012). Para que esse cuidado ocorra com dignidade é importante que os participantes desse processo como paciente, familiares e profissionais reconheçam a complexidade do cuidado paliativo em termos físicos, psicológicos, sociais e domínios espirituais.

\section{Categoria II - Prática assistencial promovida pelos enfermeiros aos pacientes em Cuidados Paliativos}

A prática assistencial relacionada ao cuidado paliativo é descrita nos trechos a seguir:

"[...]. A prática será embasada em suporte respiratório, controle de náusea, controle de dor, medidas assistenciais que não vão levar a cura mais vão trazer conforto ao paciente [...]." (ENF 9)

"A prática seria embasada em oferecer o máximo de conforto possível, manter a integridade cutânea, a higiene e manter o paciente nutrido." (ENF 12)

"Administramos as medicações prescritas para diminuição da dor, controlamos o desconforto respiratório, procuramos trabalhar junto com a família a parte psicológica e espiritual, procurando sempre envolver a família na assistência." (ENF20)

Os enfermeiros entrevistados afirmam que a prática do cuidado paliativo deve ser embasada em medidas que garantam a manutenção das necessidades humanas básicas, tais como, alívio da dor, nutrição, higiene, conforto e assistência psicológica, principalmente, ao considerar a importância da família e a valorização da dimensão espiritual no cuidar. 
Diante da exigência de oferecer um olhar atento e cauteloso ao paciente e a sua família, a equipe deve ofertar estratégias para o enfrentamento da terminalidade e continuar durante o período de "luto", especialmente o enfermeiro, por ser o profissional que lida diretamente com o paciente em cuidados paliativos (ANCP 2012; TOLEDO AP e PRIOLLI DG, 2012; PEREIRA DG, et al., 2017).

De acordo com os resultados do presente estudo, o enfermeiro apresenta-se como um pilar durante a assistência prestada a esses pacientes, devido ao tempo dispensado e a proximidade que se estabelece durante as ações de cuidado.

Exigindo desse profissional um cuidar diferenciado, prezando pela qualidade de vida e conforto dos pacientes, buscando sempre o cuidar e não o curar. Ressalta-se que os cuidados de maior destaque, referido pela maioria dos profissionais de enfermagem entrevistados para a manutenção do bem-estar do paciente foram as intervenções ligadas ao alívio da dor, como visto nos depoimentos a seguir:

"Usamos muito na nossa prática para alívio da dor as bombas de morfina prescritas pelos médicos, usamos também outras medicações extra para alívio da dor [...]." (ENF 1)

"Devemos sempre avaliar o seu nível de dor, o seu sofrimento, estar atento a mudança de decúbito, estar atento a sua alimentação, deve-se prestar uma assistência minuciosa." (ENF 6)

"[...]nossa prática é baseada, por exemplo, se o paciente estiver com dor, nós minimizamos a dor, seja com medicação injetável, compressas." (ENF 15)

Os entrevistados colocaram como uma das mais importantes práticas do cuidado paliativo as intervenções de alívio da dor, que poderá ocorrer de forma invasiva ou não. Pesquisa afirma que o tratamento para o alívio da dor deve considerar a complexidade farmacológica e fisiológica da falência de múltiplos órgãos.

Para o manejo da dor existem os opióides como a morfina, os analgésicos não opióides como a acetaminofen e as medidas não farmacológicas que envolvem massagem, musicoterapia e técnicas de relaxamento.

Salienta-se que as medidas não farmacológicas possuem menor custo, são mais seguras pelo fato não causarem efeitos adversos e podem ser facilmente providenciadas. A prevenção ou manejo da dor está associada ao melhor controle do estresse agudo e o aumento da estabilidade fisiológica (PUNTILLO et al., 2014).

Evidenciou-se nas entrevistas a importância da capacidade do enfermeiro em identificar as necessidades de conforto, destacando-se como uma das principais medidas o controle e alívio da dor. Por meio da avaliação, do seu grau de intensidade e a sua origem, para que sejam planejadas as intervenções de acordo com as necessidades do paciente, podendo ser utilizadas intervenções farmacológicas ou não.

Desse modo, é de extrema importância que o profissional proporcione a assistência baseada na humanização, respeito e acolhimento, encorajando o paciente e a família a compartilhar seus valores e objetivos de cuidado, ser sincero com seus benefícios e riscos, dar qualidade de vida, fazendo com que 0 profissional entenda o que é realmente importante para o paciente (ANDERSON EW et al., 2017).

\section{Categoria III - Relacionamento entre pacientes em cuidados paliativos, equipe multiprofissional e familiares}

Para que as intervenções sejam eficazes é primordial a construção de um relacionamento harmonioso, com respeito entre a equipe, pacientes e familiares:

"A relação se dá de forma harmoniosa. A partir do momento que a família aceita os cuidados paliativos é explicada a família o prognóstico do paciente e que não há mais chances de cura e fala da importância desses cuidados para uma morte digna com menos sofrimento[...]." (ENF 1) 
"É uma interação bem respeitosa, onde o paciente está em seus últimos momentos de vida; devemos sempre fazer nosso cuidado respeitando a dor do paciente e de sua família, tendo bastante compreensão com o paciente e seus familiares. A relação enfermeiro- paciente- família deve ser a mais horizontal possível, até na própria decisão dos cuidados paliativos em si." (ENF 7)

O resultado do presente estudo demonstra a importância da relação do enfermeiro com a família e paciente no que se refere a uma relação harmoniosa, na qual são compartilhadas informações sobre os tipos de intervenções escolhidas e o porquê destas intervenções, sempre respeitando o sofrimento da família e buscando trazer o máximo de conforto possível dentro da singularidade de cada contexto.

O cuidado paliativo é designado através das ações ofertadas por equipe multiprofissional, consiste em um cuidar voltado a atender os aspectos físicos, mentais, espirituais e sociais dos pacientes, devendo assisti-lo de forma integral. O sucesso do cuidado paliativo está relacionado à coesão e o bem-estar entre os membros da equipe (HERMES HR, LAMARCA ICA, 2013; MEHTA DH, et al., 2016).

Outro aspecto de grande importância na relação entre os membros da equipe multiprofissional, paciente e família, é a comunicação. Como os relatos a seguir demonstram:

"Toda assistência é feita a base de informação tanto entre a equipe, como entre a equipe e a família. Informamos todas as intervenções que serão feitas e as que não serão, para que os familiares não entendam que estamos negligenciando o cuidado." (ENF 12)

"[...] tentamos humanizar a assistência o máximo que podemos com o diálogo e os recursos que são oferecidos aqui no hospital." (ENF 14)

"Nós enfermeiros buscamos sempre envolver a família nesse cuidado, buscamos sempre conversar com os médicos plantonistas as questões sobre alguns procedimentos que achamos desnecessários." (ENF 20)

Nessa perspectiva, observou-se nos relatos que a equipe multiprofissional realiza o plano de intervenções em conjunto, para que seja prestado um cuidado na mesma linha, vislumbrando o mesmo objetivo; assim como a discussão dos casos do paciente com a equipe, para que sejam minimizadas as intervenções invasivas e desnecessárias. Contribuindo assim para a construção de um plano de cuidado resolutivo e integral para pacientes e familiares.

O ponto negativo observado nas falas a seguir, está relacionado ao cuidado ainda muito focado na prescrição médica e a difícil aceitação:

"Infelizmente aqui no hospital possui um cuidado muito focado no médico, voltado para prescrição médica. São eles que definem o cuidado paliativo e nós enfermeiros não participamos da discussão com a família para essa definição [...]." (ENF 16)

"Bem, como é uma prática comum, mais de difícil aceitação pelos pacientes, familiares e equipe, então nós enfermeiros procuramos ter a melhor relação possível, procurando suprir as necessidades dos pacientes e seus familiares, tentando garantir sempre uma boa assistência [...]." (ENF 4)

Por outro lado, foi relatado que em muitas ocasiões é realizado apenas o que é prescrito pelo médico, não havendo uma discussão sobre o caso do paciente para que seja realizada uma intervenção multiprofissional e mais holística para o binômio família/paciente.

A equipe paliativista deve ser constituída, no mínimo, por médicos, enfermeiros, psicólogos e assistentes sociais; diante da exigência de oferecer um olhar atento e cauteloso ao paciente e a sua família, devendo ofertar estratégias para o enfrentamento da terminalidade e continuar durante o luto (ANCP, 2012; TOLEDO AP, PRIOLLI DG, 2012; PEREIRA DG et al, 2017). A forma ideal de se paliar é através da discussão do progresso da doença e do plano de cuidado junto à equipe multiprofissional (FAN SY, 2017). 
Além disso, os enfermeiros devem ser preparados para promoverem educação em saúde, possibilitando o conhecimento sobre as patologias, identificando questões espirituais e culturais, provendo suporte físico e psicossocial, criando e implementando o plano de cuidados, assim como, sendo líderes do desenvolvimento da promoção, implementação e sustentação do trabalho multiprofissional nos cuidados paliativos (ANA, 2015). Apesar de todas estas possibilidades a serem desenvolvidas pelos enfermeiros, os resultados demonstraram que muitas vezes não há participação da enfermagem no momento em que é instituído o cuidado paliativo, bem como, na elaboração do plano de cuidados do paciente. Essa abordagem está sendo feita apenas pela classe médica, mas deveria estar sendo realizada por toda equipe multiprofissional de forma interdisciplinar.

\section{Categoria IV - Dificuldades encontradas pelos enfermeiros na assistência direcionada ao paciente em Cuidados Paliativos}

O grande desafio encontrado pelos enfermeiros entrevistados foi em relação a falta do protocolo de cuidados paliativo:

"A maior dificuldade encontrada é o momento de fechar os cuidados paliativos com a família, e pela falta de um protocolo onde muitas vezes são tiradas algumas coisas daquele paciente como dieta, sendo algo difícil essa decisão para a família." (ENF 4)

"Eu acho que ajudando e incentivando a criação de um protocolo, porque aqui na clínica o cuidado paliativo é feito de forma "perdida", confusa, de boca. Tudo é feito segundo a prescrição medica e sabemos que nós enfermeiros podemos fazer muito pelo conforto do paciente. Aqui somos mais focados apenas no alívio da dor." (ENF 8)

"A dificuldade não é em relação ao paciente nem a família, mais sim entre os profissionais, como não há uma linguagem única, uma comunicação, uma troca de informações sobre o que se deve ou não fazer por aquele paciente, o cuidado fica fragilizado." (ENF 5)

Outro fator importante observado nos resultados refere-se à sugestão da adoção de um protocolo específico para a padronização da assistência pelos enfermeiros, facilitando a intervenção de enfermagem e as visitas multidisciplinares. Um relatório de melhoria de qualidade, realizado na Universidade de Indiana nos Estados Unidos, afirma que o protocolo deve ser elaborado pela equipe multiprofissional para que haja o sucesso das intervenções e para manutenção de uma comunicação consistente e o compartilhamento de informações padrões, para que seja assegurada a continuidade do cuidado paliativo (SINHA S, et al., 2017).

Sendo assim, o uso de protocolo de cuidados para pacientes no final da vida é de extrema importância para a sistematização do cuidado de enfermagem, com a finalidade de se obter resultados eficazes de forma mais humana e com alta qualidade (SANTOS EC et al., 2016).

Outro fator observado nas falas foi a necessidade de cursos de capacitação na área de cuidados paliativos para melhorar a assistência ao paciente:

"Seria muito importante a capacitação sobre os cuidados paliativos, para poder começar a desenvolver ações de forma mais precoce com equipe multiprofissional para fechar esses cuidados e realizar ele de forma efetiva, porque aqui na clínica a paliação começa nos últimos dias de vida do paciente." (ENF 9)

"[...] buscar capacitações, seria também uma forma de contribuição prestando um cuidado mais humanizado, pois o serviço em si não está organizado para essa demanda[...]." (ENF 11)

Além da implementação do protocolo, a falta de capacitação profissional foi uma das dificuldades elencadas pelos entrevistados. Todos os participantes apresentaram especializações, mas nenhuma delas na área de cuidados paliativos. Segundo estudo qualitativo realizado no Brasil, a realização do cuidado 
paliativo pelos profissionais deve ser embasada pela filosofia paliativista. A atuação profissional é permeada, muitas vezes, por incertezas e conhecimentos insuficientes, relacionado à falta de um referencial teórico e treinamento sobre esta prática (IKEDA LHM, et al., 2017).

O presente estudo destaca que os profissionais não possuem amparo acadêmico para desenvolver cuidados ao paciente que se encontra em processo final de vida, e como alternativa desta lacuna, eles afirmam que a educação permanente seria uma importante ferramenta para melhoria da qualidade do serviço prestado. Portanto, é de suma importância que o enfermeiro esteja capacitado para realização dos cuidados paliativos, através da formação universitária em saúde ou de cursos de capacitação; que o habilitem para a realização desse cuidado específico, tão necessário aos pacientes com doenças ameaçadoras da vida, proporcionando uma assistência específica a essa clientela baseada na humanização, no respeito e acolhimento.

\section{CONCLUSÃO}

Por meio da análise, juntamente, com o aporte teórico foi possível depreender a importância dos cuidados paliativos no âmbito hospitalar na visão dos enfermeiros. Percebemos ser fundamental a capacitação e preparo destes profissionais para minimizar as possíveis dificuldades encontradas $\mathrm{n}$ processo de finitude de vida que envolvam a tríade dos cuidados: profissionais, pacientes e família. Ressaltamos a necessidade de novos estudos que busquem aprofundar o conhecimento e as experiências dos profissionais da enfermagem em cuidados paliativos para que assim possam auxiliar e embasar os conhecimentos dos profissionais de saúde em suas limitações científicas e práticas.

\section{REFERÊNCIAS}

1. ACADEMIA NACIONAL DE CUIDADOS PALIATIVOS (ANCP). Manual de cuidados paliativos. Rio de Janeiro: Diagraphic; 2012.

2. AMERICAN NURSES ASSOCIATION (ANA). Nursing: scope and standards of practice. Silver Spring, Maryland. 3rd edition, 2015; 260p

3. ANDERSON EW et al. Combining Best Practices and Patient, Caregiver, and Healthcare Provider Perspectives for Late-Life Supportive Care: LifeCourse. Journal of Nursing Administration, 2017; 47(11):551-557.

4. BALIZA MF, et al. Factors influencing intensive care units nurses in end-of-life decisions. Esc Enferm USP, 2015;49(4): 572-9.

5. BARDIN L. Análise de Conteúdo. Lisboa: Edições 70; 2011.

6. BRASIL. Ministério da saúde (MS). Conselho Nacional de Saúde. Comissão de Ética e Pesquisa. Resolução no 466/2012 sobre pesquisa envolvendo seres humanos. Brasília: DF; 2012.

7. BRITTO SMC, et al. Representação social dos enfermeiros sobre cuidados paliativos. Ver Cuid. Bucaramanga, 2015; 6(2):1062-1069.

8. FAN SY. Psychosocial Care Provided by Physicians and Nurses in Palliative Care: A Mixed Methods Study. J Pain Symptom Manage, 2017; 53(2):216-223.

9. FELIX ZC, et al. O cuidar de enfermagem na terminalidade: observância dos princípios da bioética. Rev Gaúcha Enferm, 2014;35(3)97-102.

10. FERNANDES MA, et al. Percepção dos enfermeiros sobre o significado dos cuidados paliativos em pacientes com câncer terminal. Ciênc. saúde coletiva, 2013; 18(9):2589-2596.

11. HERMES HR, LAMARCA ICA. Cuidados paliativos: uma abordagem a partir das categorias profissionais de saúde. Ciênc. saúde coletiva, 2013; 18(9): 2577-2588.

12. IKEDA LHM, et al. Dificuldades de uma equipe de enfermagem para prestar cuidados paliativos. Atas CIAIQ, $2017 ; 2$.

13. ISHIKAWA Y, et al. Family preference for place of death mediates the relationship between patient preference and actual place of death: a nationwide retrospective cross-sectional study. PLoSOne, 2013;8(3):e56848.

14. LIMA MPO, OLIVEIRA MCX. Significados do cuidado de enfermagem para familiares de pacientes em tratamento paliativo. Rev Rene, 2015;16(4):593-602.

15. MEHTA DH, et al. Building Resiliency in a Palliative Care Team: A Pilot Study. J Pain Symptom Manage, 2016; 51(3):604-8.

16. PASK S, ET AL.; A framework for complexity in palliative care: A qualitative study with patients, family carers and professionals. Palliat Med., 2018; 32(6):1078-1090. 
17. PEREIRA DG et al. Significados dos cuidados paliativos na ótica de enfermeiros e gestores da atenção primária à saúde. Revista de enfermagem UFPE online, 2017; 11(3): 1357-1364.

18. PEREIRA DG, et al. Significados dos cuidados paliativos na ótica de enfermeiros e gestores da atenção primária à saúde. Revista de enfermagem UFPE online, 2017;11(3):1357-1364.

19. PUNTILLO et al. Palliative care in the ICU: relief of pain, dyspnea, and thirst--a report from the IPAL-ICU Advisory Board. Intensive Care Med., 2014; 40(2): 235-248.

20. QUEIRÓS PJP. Cuidar: da condição de existência humana ao cuidar integral profissionalizado. Rev. Enf. Ref, 2015; ser IV (5):139-146.

21. QUEIROZ TA, et al. Cuidados Paliativos ao idoso na terapia intensiva: olhar da equipe de enfermagem. Texto contexto Enferm, 2018; 27(1):e1420016.

22. SANTOS EC, et al. Validation of a nursing care protocol for patients undergoing palliative care. Acta Paul. Enferm., 2016; 29(4): 363-373.

23. SILVEIRA NR, et al. Cuidado paliativo e enfermeiros de terapia intensiva: sentimentos que ficam. Rev. Bras. Enferm, 2016; 69(6):1074-1081.

24. SINHA S, et al. Development of a Protocol for Successful Palliative Care Consultation in Population of Patients Receiving Mechanical Circulatory Support. Send to J Pain Symptom Manage, 2017; 54(4):583-588.

25. TOLEDO AP, PRIOLLI DG. Cuidados no fim da vida: o Ensino Médico no Brasil. Rev. Bra de Educação Médica, 2012;36(1):109-17.

26. VASQUES TCS, et al. Percepção dos trabalhadores de enfermagem acerca do cuidado ao paciente em terminalidade no ambiente hospitalar. Texto Contexto Enferm, 2016; 25(3): e0480014.

27. WORLD HEALTH ORGANIZATION (WHO). Cuidados paliativos: nota descritiva [Internet]. WHO, 2017 [citado 2018 maio 02]. Disponível em: http://www.who.int/mediacentre/factsheets/fs 402/en/ 